\title{
Determination of active site of Lysine-specific cysteine proteinase (Lys-gingipain) by use of a Porphyromonas gingivalis plasmid system
}

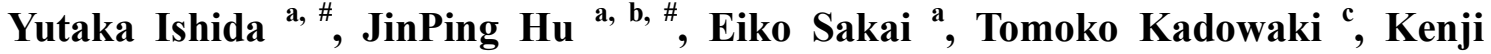

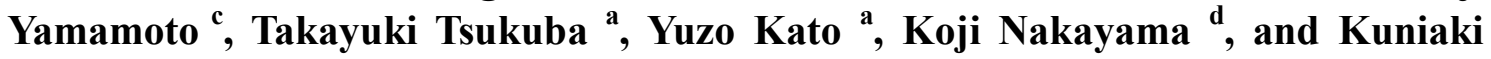 \\ Okamoto $^{\mathrm{a}, *}$
}

\begin{abstract}
${ }^{a}$ Department of Oral Pathopharmacology, Unit of Basic Medical Sciences, Medical and Dental Sciences, and ${ }^{\mathrm{d}}$ Department of Oral Microbiology and Oral Infection, Unit of Molecular Microbiology and Immunology, Infection Research, Graduate School of Biomedical Sciences, Nagasaki University, Nagasaki 852-8588; ${ }^{\mathrm{b}}$ Stomatological Hospital of Jilin University, Changchun 130041, PR China; ${ }^{\mathrm{c}}$ Department Pharmacology, Graduate School of Dental Sciences, Kyushu University, Higashi-ku, Fukuoka 812-8582, Japan
\end{abstract}

\footnotetext{
* To whom correspondence should be addressed to: Kuniaki Okamoto, Department of Oral Pathopharmacology, Unit of Basic Medical Sciences, Medical and Dental Sciences, Graduate School of Biomedical Sciences, Nagasaki University, Japan

Tel: +81-95-819-7653, Fax: +81-95-819-7655

E-mail: k-oka@nagasaki-u.ac.jp (K. Okamoto)
}

\# These authors contributed equally to this work.

The abbreviations used: Amp; ampicillin, Boc; t-butyloxycarbonyl; E. coli; Escherichia coli, Gm; gentamicin, Kgp; Lys-gingipain, MCA; 4-methyl-7-coumaryl-amide, PAGE; polyacrylamide gel electrophoresis, PCR; polymerase chain reaction, P. gingivalis; Porphyromonas gingivalis, Rgp; Arg-gingipain, SDS; sodium dodecyl sulfate, Tc; tetracycline, Z; Benzyloxycarbonyl

\begin{abstract}
Porphyromonas gingivalis, a major etiological bacterium of periodontal diseases, produces a unique lysine-specific cysteine proteinase (Lys-gingipain, Kgp) implicated in the virulence of this organism. Our observations show the expression of a catalytically active recombinant Kgp in a $P$. gingivalis Kgp-null mutant and the restoration of its functions by use of a shuttle plasmid vector stable in $P$. gingivalis. The Kgp-expressing mutant exhibited a similar catalytic activity to that of the wild-type strain. This mutant also restored the ability to form black-pigmented colonies on blood agar plates and to generate a 19-kDa hemoglobin receptor protein responsible for hemoglobin binding. In order to establish the importance of the active-site Cys residue and elucidate its role in bacterial black pigmentation we constructed three Kgp mutants with changed potential active-site Cys residues. The cells expressing a single mutation (C476A) showed the high Kgp activity and the black pigmentation. In contrast, the cells expressing the single mutant (C477A) and the double mutant (C476A/C477A) exhibited neither Kgp activity nor black pigmentation. These results indicate that the 477th Cys residue is essential for both the Kgp activity and the black pigmentation of $P$. gingivalis.
\end{abstract}

Key words: Arg-gingipain, Lys-gingipain, Periodontal diseases, Porphyromonas gingivalis, recombinant expression 


\section{Introduction}

Porphyromonas gingivalis is one of the most important pathogenic bacteria in progressive periodontal disease $\mathrm{e}^{1-7}$ and it produces a unique class of cysteine proteinases in both cellassociated and secretory forms, now termed Arggingipain (Rgp) and Lys-gingipain (Kgp). 6, 8-17 Rgp is encoded by two $\operatorname{rgp}$ genes ( $r g p A$ and $\operatorname{rgp} B),{ }^{18-23}$ whereas $\mathrm{Kgp}$ is encoded by a single kgp gene. ${ }^{25-27}$ The kgp gene encodes a polyprotein with 1732 amino acid residues, which consists of four domains: a signal peptide, a prosequence, a proteinase, and C-terminal adhesin domains. ${ }^{26,24,}$ 27 The C-terminal adhesin domain of kgp is highly homologous to that of $\operatorname{rgp} A$, although the prosequence and proteinase domains have no sequence similarity. The C-terminal adhesin domain, which is thought to be involved in hemagglutination, comprises three subdomains. A 19-kDa subdomain, termed HbR (also Hgp15), is thought to be a hemoglobin-binding receptor and thereby participates in heme accumulation. On the other hand, the $\operatorname{rgp} A$ gene encodes a polyprotein with 1704 amino acid residues, while it also consists of four domains. The $\operatorname{rgp} B$ gene is almost identical to the $\operatorname{rgp} A$ gene, except for the fact that it lacks most of the C-terminal adhesin domain of $\operatorname{rgp}^{21}$

We previously found the Kgp-null mutant to form less black-pigmented colonies on blood agar plates and failed to express a $19-\mathrm{kDa} \mathrm{HbR}$ protein, probably due to a disruption of the processing of its precursor proteins, such as RgpA, Kgp, and HagA, to generate HbR in the Kgp-null mutant. ${ }^{25}$ Given that colonial black pigmentation is caused by the accumulation of heme derivatives on the bacterial cell surface, ${ }^{29}$ the disruption of $\mathrm{HbR}$ generation is likely to account for the nonpigmentation of the Kgp-null mutant. In addition to a disruption of the generation of $\mathrm{HbR}$, we also showed this Kgp-null mutant to decrease the ability to degrade fibrinogen. ${ }^{25}$ These results provide evidence suggesting that $\mathrm{Kgp}$ is associated with hemagglutination, hemoglobin binding, heme accumulation, and bleeding tendency in the periodontal pockets.

Recently, Sztukowska et $a l^{30}$ reported the C-terminal domain of Kgp to be necessary for the assembly and expression of catalytically active Kgp. They also found that the expression of the Kgp proteinase domain alone resulted in the expression of Kgp with a weak activity. In the present study, we constructed a shuttle plasmid vector harboring the kgp gene lacking its Cterminal adhesin domain and successively expressed in the Kgp-null mutant. The mutant expressing recombinant $\mathrm{Kgp}$ restored the enzymatic activity comparable to that of the wildtype strain and the ability to form blackpigmented colonies on blood agar plates. Using this shuttle plasmid vector system, we also constructed three Kgp mutants with changed potential active-site Cys residues and demonstrate that Cys residue $\left(477^{\text {th }} \mathrm{Cys}\right)$ at its potential active site is therefore essential for both the Kgp activity and the black pigmentation of $P$. gingivalis.

\section{Materials and methods}

\subsection{Reagents}

Benzyloxycarbonyl (Z)-Phe-Arg-4-methyl-7counmarylamide (MCA) and $t$-butyloxycarbonyl (Boc)-Val-Leu-Lys-MCA as synthetic substrates were purchased from the Peptide Institute (Osaka, Japan).

\subsection{Bacteria and culture conditions}

P. gingivalis ATCC33277 was used as a wild-type strain. The Kgp-deficient mutant (KDP129) has been described previously. ${ }^{25}$ Both the wild-type strain and the KDP129 were grown under anaerobic conditions $(10 \% \mathrm{CO} 2,10 \% \mathrm{H} 2,80 \%$ $\mathrm{N} 2$ ) at $37^{\circ} \mathrm{C}$ in enriched BHI broth (containing, per liter, $37 \mathrm{~g}$ of brain heart infusion (Difco), $5 \mathrm{~g}$ of yeast extract (Difco), $1 \mathrm{~g}$ of cysteine, $5 \mathrm{mg}$ of hemin, and $1 \mathrm{mg}$ of vitamin $\mathrm{K} 1$ ), on tryptic soy agar (TS) (containing, per liter, $40 \mathrm{~g}$ of TryptoSoya agar (Nissui, Tokyo, Japan), $5 \mathrm{~g}$ of brain 
heart infusion (Difco), $1 \mathrm{~g}$ of cysteine, $5 \mathrm{mg}$ of hemin, and $1 \mathrm{mg}$ of vitamin $\mathrm{K} 1$ ) and blood agar plate (TS plate containing 5\% sheep blood). L broth (containing, per liter, $10 \mathrm{~g}$ of tryptone (Difco), $5 \mathrm{~g}$ of yeast extract (Difco), and $5 \mathrm{~g}$ of sodium chloride) was used to grow the E. coli cells. Tetracycline (Tc) $(1 \mu \mathrm{g} / \mathrm{ml})$, gentamicin (Gm) $(100 \mu \mathrm{g} / \mathrm{ml})$, and ampicillin (Amp) (200 $\mu \mathrm{g} / \mathrm{ml}$ ) were added to the medium when necessary.

\subsection{Plasmid construction}

A shuttle vector stably existing in $P$. gingivalis, pYKP028, was a kind gift from Dr. Yumi Kumagai in Nippon Dental University, Japan. ${ }^{31}$ All plasmids were amplified by polymerase chain reaction (PCR) and pNKV encoding the partial kgp gene was used as a template. ${ }^{24}$ Briefly, the $K p n I$ restriction enzyme sites were designated as the 5'- and 3'-region and the Kgp gene including the promoter region, signal-peptide, pro-peptide, and mature proteinase domain were amplified. After being digested by $K p n I$, these fragments were collected and subcloned into the KpnI site of pYKP028 dephosphorylated by bovine alkaline phosphatase. These plasmids were then introduced into $P$. gingivalis. A mutation occurred by site-directed mutagenesis using the primers noted below: The primer used: Kgp-s; 5'AATGGTACCTATCATATCAG-3' and Kgp-as; 5'-

GCGGTACCTTAACGTACATCGTTTGCAGGT3' for NOP003 (pNOP003), 5' GCCTGTGTTACAGCTCAATTCGATT-3' and DM-as; 5'-GTTCCCAATAGCTAAGAAGT-3' for $\quad$ 476A (pNOP031), 5'TGCGCTGTTACAGCTCAATTCGATT-3' and DM-as for C477A (pNOP021), 5' GCCGCTGTTACAGCTCAATTCGATT-3' and DM-as for C476A C477A (pNOP011).

Mobilization from $E$. coli to $P$. gingivalis ---- The procedure for the mobilization of plasmids from $E$. coli to $P$. gingivalis has been previously described. $^{32}$ One tenth volume of an early exponential phase culture of $E$. coli SM10גpir (1 $\times 10^{8}$ cells $/ \mathrm{ml}$ ) harboring only pYKP028 as a control, pNOP003, pNOP011, pNOP021, and pNOP031 was mixed with one tenth volume of the early exponential phase culture of $P$. gingivalis KDP129 (Kgp-deficient mutant) $(1 \times$ $10^{9}$ cells $/ \mathrm{ml}$ ), and the mixed cells were then collected by centrifugation. The mixed pellet was suspended in $1 \mathrm{ml}$ of pre-warmed enriched BHI broth, spotted on enriched TS plates and then incubated anaerobically at $37^{\circ} \mathrm{C}$ for 1 day. The cells on the plates were collected, suspended in 1 $\mathrm{ml}$ of pre-warmed BHI broth, spread on the TS plates containing $\mathrm{Tc}$ and $\mathrm{Gm}$ and incubated anaerobically at $37^{\circ} \mathrm{C}$ for 7 days. After selection, we obtained about 50-100 colonies in all mutants.

\subsection{Proteinase activity}

The proteolytic activities of Rgp and Kgp were determined with Z-Phe-Arg-MCA and Boc-ValLeu-Lys-MCA as described previously. ${ }^{16,}{ }^{17}$ Briefly, the reaction mixture $(1 \mathrm{ml})$ contained various amounts of cell extracts or the culture supernatants of an overnight culture, $10 \mu \mathrm{M}$ of each synthetic substrate, and $5 \mathrm{mM}$ cysteine in 20 $\mathrm{mM}$ sodium phosphate buffer $(\mathrm{pH}$ 7.5). After incubation at $40^{\circ} \mathrm{C}$ for $10 \mathrm{~min}$, the reaction was terminated by adding $1 \mathrm{ml}$ of $10 \mathrm{mM}$ iodoacetic acid ( $\mathrm{pH}$ 5.0), and the released MCA was then measured at $460 \mathrm{~nm}$ (excitation at $380 \mathrm{~nm}$ ) on a fluorescence spectrophotometer. As wild-type (ATCC33277) and KDP129 introduced only a vector (pYKP028) have Kgp and Rgp activities as strong as those of wild-type and KDP129, respectively (data not shown), the wild-type and KDP129 strains were therefore used in this study as a control.

\subsection{Gel electrophoresis and immunoblot analysis}

Sodium dodecyl sulfate-polyacrylamide gel electrophoresis (PAGE) was performed according to the method of Laemmli. ${ }^{33}$ The proteinase inhibitors leupeptin and $p$-toluenesulfonyl-L- 
phenylalanine chloromethyl ketone were added to a solubilizing buffer to avoid proteolysis by endogeneous proteinases. For immunoblotting, protein on SDS gels was electrophoretically transferred to nitrocellulose membranes according to the method of Towbin et al.. ${ }^{34}$ The blotted membranes were immunostained with the polyclonal antibodies immunoreacting to 40 amino acid sequences of the mature Kgp-Nterminus, which was both Rgp and Kgp purified ${ }^{16}$, 17 or $19-\mathrm{kDa}$ hemoglobin receptor protein purified. $^{35}$

\subsection{Sequencing}

Nucleotide sequencing was determined by automated DNA sequencing (ABI PRISM 310 Genetic Analyzer, Applied Biosystems, Foster, CA) using the BigDye Terminator Cycle Sequencing Kit (Applied Biosystems).

\subsection{Statistical analysis}

The quantitative data are presented as the mean \pm S.D. The statistical significance of differences between the mean values was assessed by Student's $t$-test. $p$ values of $<0.01$ were considered statistically significant.

\section{Results}

\subsection{Functional expression of KGP}

To construct the Kgp expression system, a PCRamplified DNA product containing the promoter region, signal peptide, propeptide and proteinase domain of $k g p$ was inserted into the $P$. gingivalis shuttle plasmid vector pYKP028 which was able to be stably maintained in both $E$. coli and $P$. gingivalis (Fig. 1A). The resulting plasmid pNOP003 was mobilized from E. coli SM10גpir into the $P$. gingivalis kgp mutant (KDP129). KDP129 harboring pNOP003, which is designated as NOP003, was examined to determine the expression levels of both the Kgp and Rgp activities using the respective synthetic substrates (Fig. 1B and D). Although KDP129 had no Kgp activity in either the cell lysate or the culture supernatant, NOP003 clearly restored its activity in both fractions. The expression of Kgp and Rgp at protein levels was also determined by a Western blot analysis using antibodies specific for Kgp and antibodies immunoreacting with the catalytic domains of both Rgp and Kgp (Fig. 1C and $\mathrm{E}$ ). The 51-kDa proteinase domain of Kgp, like the $44-\mathrm{kDa}$ proteinase domain of Rgp, was restored in NOP003.

\subsection{Recovery of black pigmentation and processing of $\mathrm{HbR}$}

We previously demonstrated that Kgp deficiency resulted in the lack of colonial black pigmentation onto blood agar plates and the impaired production of $\mathrm{HbR} .{ }^{25}$ To determine whether NOP003 is able to form black pigmented colonies, it was incubated on the blood agar plate (Fig. 2A). Whereas KDP129 exhibited little black pigmentation, NOP003 formed more black pigmented colonies than those formed by the wild-type strain. Consistently, the ability to produce the 19-kDa HbR protein, which was not observed with KDP129, was restored in NOP003 to the level of that of the wild-type strain (Fig. $2 \mathrm{~B})$. These results indicate that the recombinant Kgp expressed in a form lacking the C-terminal adhesin domain has normal catalytic activity, thus resulting in the restoration of the bacterial functions caused by Kgp deficiency.

\subsection{Identification of active sites of KGP}

We have previously suggested that an active-site Cys residue of $\mathrm{Rgp}$ is $471^{\text {st }} \mathrm{Cys}$, based on the sequence similarity of the active site region to that of clostripain. ${ }^{22}$ This notion is supported by the results obtained by active-site mapping experiments using $\mathrm{N \alpha}-\left[{ }^{3} \mathrm{H}\right]$ acetyllysine chloromethyl ketone ${ }^{36}$ and peptidyl chloromethanes. ${ }^{37}$ Given that the sequence similarity of the active-site regions between Rgp and $\mathrm{Kgp}$, the active site Cys residue of $\mathrm{Kgp}$ is assumed to be $476^{\text {th }}$ and/or $477^{\text {th }}$ Cys. To 
determine which Cys residues are essential for the $\mathrm{Kgp}$ activity, we constructed and expressed Kgp mutants with changed these residues (C476A, C477A, and C476A/C477A, termed NOP031, NOP021, and NOP011, respectively) in KDP129 (Fig. 3A). A Western blot analysis with antibodies specific to Kgp revealed that all the three mutants were produced at an apparent molecular mass of $51 \mathrm{kDa}$, which is identical with that of the wildtype strain (Fig. 3B). One of the single mutants, NOP031, showed a significant Kgp activity in both the cell lysate and the culture supernatant, although their levels were lower than those of the wild-type strain (Fig. 3C). In contrast, the single mutant NOP021 and the double mutant NOP011 completely lost the Kgp activity. Consistent with these results, whereas the former single mutant formed black-pigmented colonies on a blood agar plate (Fig. 4) and generated the 19-kDa HbR protein (data not shown), the latter single mutant and the double mutant showed no colonial pigmentation and no production of HbR protein. On the other hand, the protein and activity levels of Rgp in all of these mutants were not significantly different from those of the wild-type strain (Fig. 5). These results indicate that the $477^{\text {th }}$ Cys residue is required for the full activity of $\mathrm{Kgp}$, but that the substitution of the active-site Cys residues of $\mathrm{Kgp}$ does not affect the expression of Rgp.

\section{Discussion}

The $P$. gingivalis sod gene encoding superoxide dismutase (SOD) has been shown to be expressed as an enzymatically active enzyme in E. coli. ${ }^{38}$ Despite extensive efforts, however, attempts to generate recombinant $\mathrm{Rgp}$ and $\mathrm{Kgp}$ in E. coli or baculovirus have so far proven to be unsuccessful. $^{22,} 39,40$ Given that SOD is a cytoplasmic protein, whereas Rgp and Kgp are cell surface and extracellular proteins, the success may depend on the cellular localization of individual proteins in $P$. gingivalis. Pavloff et al. ${ }^{39}$ successfully expressed $r g p B$ in yeast and obtained the catalytically active enzyme, although its activity was lower than that of Rgp from the wildtype strain. These authors also demonstrated the importance of the truncation at the $\mathrm{N}$-terminus for its activation. The ability of yeast to add carbohydrate moieties to proteins may serve for the correct folding of Rgp. More recently, the Cterminal adhesin domain of Kgp was shown to be necessary for the expression of the active enzyme. ${ }^{30}$ They also suggested that the C-terminal domain is essential for the proper processing of this multiprotein complex and the assembly on the cell surface. In this study, we constructed wildtype Kgp and its mutants with changed active-site Cys residues using a shuttle plasmid containing kgp derivatives without replacement by homologous recombination. When the shuttle plasmid containing the kgp gene lacking the Cterminal adhesin domain was introduced into the Kgp-null mutant by electroporation, we could not obtain any transformants, probably due to the plasmid size. We therefore introduced the plasmid into the Kgp-null mutant by mobilization from $E$. coli to $P$. gingivalis with the aid of RP4 helper plasmid in the donor $E$. coli. The resulting strain NOP003, the kgp mutant harboring the plasmid pNOP003, exhibited 53-77\% of the Kgp activity of the wild-type parent in the cell extract and culture supernatant, thus indicating that the Cterminal adhesin domain is not necessarily required for the correct folding of $\mathrm{Kgp}$ and the expression of its activity. In addition, the expression of $\operatorname{Rgp}$ at activity and the protein levels in the cells did not significantly change by the expression of the wild-type Kgp and its mutants, thus suggesting the generation of various bacterial proteins that are processed by Rgp to not be affected by whether Kgp is expressed or not.

Our previous study with Kgp-null mutants ${ }^{25}$ indicated that Kgp plays an important role in heme accumulation and black pigmentation. As the C-terminal amino acid residue of $\mathrm{HbR}$ is Lys, ${ }^{23-25,39}$ it is likely that $\mathrm{HbR}$ 
is processed by $\mathrm{Kgp}$, thereby resulting in its binding to hemoglobin. Consequently, the bacterium acquires heme from hemoglobin, which is essential for its proliferation and growth. The kgp mutant harboring pNOP003 restored the ability to form black-pigmented colonies on the blood agar plate and to generate the normaly processed $\mathrm{HbR}$, thus indicating that $\mathrm{Kgp}$ is directly involved in these events in the bacterium.

Nishikata and Yoshimura determined the active site of Rgp by using a labeling method of active sites with $\mathrm{N} \quad \alpha$-[3H]acetyllysine chloromethyl ketone. ${ }^{36}$ The labeled active site peptide isolated by high performance liquid chromatography was ${ }^{468}$ Asp-Val-Ala-Cys-ValAsn $-{ }^{474}$ Gly. The fact that the sequence resembled the active site structure of the arginine-specific cysteine proteinase clostripain from $C$. histriticum thus confirmed that the $471^{\text {st }}$ Cys residue is the active site of Rgp. Based on the alignment with the sequence, an active site region of Kgp was therefore predicted to be ${ }^{474} \mathrm{Gly}$-Asn-Cys-Cys-ValThr- ${ }^{480}$ Ala. Since two Cys residues are present in this region, it appears to be impossible to determine which Cys is an active site of $\mathrm{Kgp}$ using the labeling method. Therefore, to identify which Cys is essential for the catalytic activity of Kgp, we constructed a mutated kgp gene with a base substitution at the Cys residues on the shuttle plasmid using site-directed mutagenesis and then introduced it into KDP129. The double mutant (C476A/C477A) and one of two single mutants (C477A) showed no Kgp activity, although a distinct immunoreactive protein band with antibodies to Kgp was detected at a similar molecular size. On the contrary, another single mutant $(\mathrm{C} 476 \mathrm{~A})$ retained about $50 \%$ of the Kgp activity found in the cell lysate and the culture supernatant of the wild-type strain. These results indicate, for the first time, that the $477^{\text {th }} \mathrm{Cys}$ residue is essential for the expression of $\mathrm{Kgp}$ activity.

In conclusion, we herein observed the expression of an active $\mathrm{Kgp}$ from a plasmid possessing the kgp gene in $P$. gingivalis. The $P$. gingivalis kgp mutant expressing the recombinant Kgp showed colonial black pigmentation and a normal processing of $\mathrm{HbR}$. Using this plasmid system, we demonstrated that ${ }^{477} \mathrm{Cys}$ of $\mathrm{Kgp}$ was able to function as an active site. This expression system is therefore considered to be useful to elucidate the mechanisms of transport for periplasm proteins, outer membrane proteins, and secretary proteins in $P$. gingivalis.

\section{Acknowledgements}

This work was supported in part by a Grant-inAid for Scientific Research from the Ministry of Education, Science and Culture, Japan. We thank Dr. Y. Kumagai (Nippon Dental University School of Dentistry) for kindly giving us the $E$. coli-P. gingivalis shuttle vector plasmid pYKP028. 


\section{REFERENCES}

1. Mayrand D, Holt SC. Biology of asaccharolytic blackpigmented Bacteroides species. Microbiol Rev 1988; 52: 134-52.

2. Moore WEC, Holdeman LV, Smibert RM, Hash DE, Burmeister JA, Ranney RR. Bacteriology of severe periodontitis in young adult humans. Infect Immun 1982; 38: $1137-48$.

3. Zambon JJ, Reynolds HS, Slots J. Black-pigmented Bacteroides spp. in the human oral cavity. Infect Immun 1981; 32: 198-203.

4. Marsh PD, McKee AS, McDermid AS, Dowsett AB. Ultrastructure and enzyme activities of a virulent and an avirulent variant of Bacteroides gingivalis W50. FEMS Microbiol Lett 1989; 50: 181-5.

5. Grenier D, Mayrand D. Selected characteristics of pathogenic and nonpathogenic strains of Bacteroides gingivalis. J Clin Microbiol 1987; 25: 738-40.

6. Birkedal-Hansen H, Taylor RE, Zambon JJ, Barwa PK, Neiders ME. Characterization of collagenolytic activity from strains of Bacteroides gingivalis. J Periodont Res 1988; 23: 258-64.

7. Sundqvist G, Carlsson J, Hänström L. Collagenolytic activity of black-pigmented Bacteroides species $J$ Periodont Res 1987; 22: 300-6.

8. Otsuka M, Endo J, Hinode D, Nagata A, Maehara R, Sato, M, Nakamura R. Isolation and characterization of protease from culture supernatant of Bacteroides gingivalis. $J$ Periodont Res 1987; 22: 491-8.

9. Sorsa T, Uitto V-J, Suomalainen K, Turto H, Lindy S. A trypsin-like protease from Bacteroides gingivalis: partial purification and characterization. J Periodont Res 1987; 22: $375-80$.

10. Smalley JW, Birss AJ, Shuttleworth CA. The degradation of type I collagen and human plasma fibronectin by the trypsin-like enzyme and extracellular membrane vesicles of Bacteroides gingivalis W50. Archs Oral Biol 1988; 33: 323-9.

11. Grenier D. Inactivation of human serum bactericidal activity by a trypsinlike protease isolated from Porphyromonas gingivalis. Infect Immun 1992; 60: 18547.

12. Chen Z, Potempa J, Polanowski A, Renvert S, Wikström M, Travis J. Stimulation of proteinase and amidase activities in Porphyromonas (Bacteroides) gingivalis by amino acids and dipeptides. Infect Immun 1991; 59: 284650.
13. Scott CF, Whitaker EJ, Hammond BF, Colman RW. Purification and characterization of a potent $70 \mathrm{kDa}$ thiol lysyl-proteinase (Lys-gingivain) from Porphyromonas gingivalis that cleaves kininogens and fibrinogen. $J$ Biol Chem 1993; 268: 7935-42.

14. Curtis MA, Ramakrishnan M, Slaney M. Characterization of the trypsin-like enzymes of Porphyromonas gingivalis W83 using a radiolabelled active-site-directed inhibitor. $J$ Gen Microbiol 1993; 139: 949-55.

15. Chen Z, Potempa J, Polanowski A, Wikstrom M, Travis J. Purification and characterization of a $50-\mathrm{kDa}$ cysteine proteinase (gingipain) from Porphyromonas gingivalis. $J$ Biol Chem 1992; 267: 18896-901.

16. Kadowaki T, Yoneda M, Okamoto K, Maeda K, Yamamoto K. Purification and characterization of a novel argininespecific cysteine proteinase (argingipain) involved in the pathogenesis of periodontal disease form the culture supernatant of Porpyhromonas gingivalis. J Biol Chem 1994; 269: 21371-8.

17. Abe N, Kadowaki T, Okamoto K, Nakayama K, Ohishi M, Yamamoto K. Biochemical and functional properties of lysine-specific cysteine proteinase (Lys-gingipain) as a virulence factor of Porphyromonas gingivalis in periodontal disease. J Biochem 1998; 123: 305-12.

18. Gallaghler A, Aduse-opoku J, Rangarajan M, Slaney JM, Curtis MA. Glycosylation of the Arg-gingipain of Porphyromonas gingivalis and comparison with glycoconjugate structure and synthesis in other bacteria. Curr Protein Pept Sci 2003; 4: 427-41.

19. Mikolajczyk-Pawinska J, Kordula T, Pavloff N, Pemberton PA, Chen WCA, Travis J, Potempa J. Genetic variation of Porphyromonas gingivalis genes encoding gingipains, cysteine proteinases with arginine or lysine specificity. Biol Chem 1998; 379: 205-11.

20. Nakayama K, Kadowaki T, Okamoto K, Yamamoto K. Construction and characterization of arginine-specific cysteine proteinase (Arg-gingipain)-deficient mutants of Porphyromonas gingivalis. J Biol Chem 1995; 270: 23619-26.

21. Nakayama K. Domain-specific rearrengement between the two Arg-gingipain-encoding genes in Porphyromonas gingivalis: possible involvement of nonreciprocal rcombination. Microbioil Immunol 1997; 41: 185-96.

22. Okamoto K, Misumi $\mathrm{Y}$, Kadowaki T, Yoneda M, Yamamoto K, Ikehara Y. Structural characterization of argingipain, novel arginine-specific cysteine proteinase as a major periodontal pathogenic factor from 
Porphyromonas gingivalis. Arch Biolchem Biophys 1995; 316: $917-25$.

23. Pavlof N, Potempa J, Pike RN, Prochaka V, Kiefer MC, Travis J, Bar PJ. Molecular cloning and structural characterization of the Arg-gingipain proteinases from Porphyromonas gingivalis. J Biol Chem 1995; 270: 100710.

24. Okamoto K, Kadowaki T, Nakayama K, Yamamoto K. Cloning and sequencing of the gene encoding a novel lysspecific cysteine proteinase (Lys-gingipain) in Porphyromonas gingivalis; Structural rlationship with the arginine-specific cysteine proteinase (Arg-gingipain). $J$ Biochem 1996; 120: 398-406.

25. Okamoto K, Nakayama K, Kadowaki T, Abe N, Ratnayake DB, Yamamoto K. Involvement of a lysine-specific cysteine proteinase in hemoglobin adsorption and heme accumulation by Porphyromonas gingivalis. J Biol Chem 1998; 273: 21225-315.

26. Kadowaki T, Nakayama K, Okamoto K, Abe N, Baba A, Shi Y, Ratnayake DB, Yamamoto K. Porphyromonas gingivalis proteinases as virulence determinants in progression of periodontal diseases. J Biochem 2000; 128: 153-9.

27. Pike R, McGraw W, Potempa J, Travis J. Lysine- and arginine-specific proteinases from Porphyromonas gingivalis. Isolation, characterization, and evidence for the existence of complexes with hemagglutinins. J Biol Chem 1994; 269: 406-11.

28. Kadowaki T, Nakayama K, Yoshimura F, Okamoto K, Abe N, Yamamoto K. Arg-gingipain Acts as a Major Processing Enzyme for Various Cell Surface Proteins in Porphyromonas gingivalis. J Biol Chem 1998; 273: 29072-6.

29. Shah HN, Gharbia SE. Lysis of erythrocytes by the secreted cysteine proteinase of Porphyromonas gingivalis W83. FEMS Microbiol Lett 1989; 61: 213-8.

30. Sztukowska M, Sroka A, Bugno M, Banbula A, Takahashi Y, Pike RN, Genco CA, Travis J, Potempa J. The Cterminal domains of the gingipain $\mathrm{K}$ polyprotein are necessary for assembly of the active enzyme and expression of associated activities. Mol Microbiol 2004; 54: 1393-408.

31. Kumagai Y, Yajima A, Konishi K. Peptidase activity of dipeptidyl aminopeptidase IV produced by Porphyromonas gingivalis is important but not sufficient for virulence. Microbiol Immunol 2003; 47: 735-43.

32. Shoji M, Naito M, Yukitake H, Sato K, Sakai E, Ohara N,
Nakayama K. The major structural components of two cell surface filaments of Porphyromonas gingivalis are matured through lipoprotein precursors. Mol Microbiol 2004; 52: 1513-25.

33. Laemmli UK. Cleavage of structural proteins during the assembly of the head of bacteriophage T4. Nature 1970; 227: $680-5$.

34. Towbin H, Staehelin T, Gordon J. Electrophoretic transfer of proteins from polyacrylamide gels to nitrocellulose sheets: procedure and some applications. Proc Natl Acad Sci USA 1979; 76: 4350-4.

35. Nakayama K, Ratnayake DB, Tsukuba T, Kadowaki T, Yamamoto K, Fujimura S. Haemoglobin receptor protein is intragenically encoded by the cysteine proteinaseencoding genes and the haemagglutinin-encoding gene of Porphyromonas gingivalis. Mol Microbiol (1998); 27: 5161.

36. Nishikata M, Yoshimura F. Active site structure of a hemagglutinating protease from Porphyromonas gingivalis: similarity to clostripain. Biochem. Mol Biol Int 1995; 37: 547-53.

37. Potempa J, Pike R, Travis J. Titration and mapping of the active site of cysteine proteinases from Porphyromonas gingivalis (gingipains) using peptidyl chloromethanes. Biol Chem 1997; 378: 223-30.

38. Nakayama K. Rapid viability loss on exposure to air in a superoxide dismutase-deficient mutant of Porphyromonas gingivalis. J Bacteriol 1994; 176: 1939-43.

39. Aduse-Opoku J, Muir J, Slaney JM, Rangarajan M, Curtis MA. Characterization, Genetic Analysis, and Expression of a Protease Antigen (PrpRI) of Porphyromonas gingivalis W50. Infect Immun 1995; 63: 4744-54.

40. Pavloff N, Pemberton PA, Potempa J, Chen WA, Pike RN, Prochazka V, Kiefer MC, Travis J, Barr PJ. Molecular cloning and characterization of Porphyromonas gingivalislysine-specific gingipain. J Biol Chem 1997; 272: $1595-600$. 
Figure legends

Fig. 1 - Characterization of the recombinant Kgp expression mutant (NOP003) compared with wild-type $P$. gingivalis (ATCC33277). (A); A schematic drawing of the domain structures of NOP003 and ATCC33277. SP; Signal Peptide, Pro; Pro-region, nGP44 and cGP44; N-terminal and $\mathrm{C}$-terminal region of $\mathrm{Kgp}$ hemagglutinin domain. K; restriction enzyme site for KpnI. (B) and (C); The activity (B) and protein (C) levels of $\mathrm{Kgp}$ in the cell lysate and culture supernatant fractions of NOP003 were compared with those of ATCC33277 and Kgp-null mutant (KDP129) by activity measurements with Boc-Val-Leu-LysMCA as a substrate and a Western blot analysis with antibodies specific to Kgp, respectively. 2.5 $\mu \mathrm{g}$ of cell lysate and $5 \mu \mathrm{g}$ of the supernatant were applied in these experiments. (D) and (E); The activity (D) and protein (E) levels of Rgp in the cell lysate and culture supernatant fractions of NOP003 were compared with those of ATCC33277 and Kgp-null mutant (KDP129) by activity measurements with Z-Phe-Arg-MCA as a substrate and a Western blot analysis with antibodies reacting with both $\mathrm{Rgp}$ and $\mathrm{Kgp}$, respectively. $1 \mu \mathrm{g}$ of cell lysate and $2 \mu \mathrm{g}$ of the supernatant were applied in these experiments. The white and black bars indicate the cell lysate and the culture supernatant, respectively. The data are the mean \pm S.D. of values from 5 independent experiments. *, $p<0.01$ for the indicated comparisons.

Fig. 2 - Restoration of the abilities to form black-pigmented colonies and to generate $\mathrm{HbR}$ protein in NOP003. (A) Colonial black pigmentation by ATCC33277, KDP129 and NOP003 that were anaerobically incubated at $37^{\circ} \mathrm{C}$ for 5 days onto a laked sheep blood agar plate. (B) Western blot analysis of the cell lysate and supernatant fractions from each bacterial strain with antibodies specific for HbR. $2 \mu \mathrm{g}$ of cell lysate and the supernatant were applied in these experiments.
Fig. 3 - Characterization of Kgp mutants with changed active-site Cys residues in KDP129. (A) A sequence alignment of the active site regions of $\mathrm{Kgp}$ in ATCC33277, single mutants NOP031 (C476A) and NOP021 (C477A), and the double mutant NOP011 (C476A/C477A). (B) A Western blot analysis of the cell lysate and culture supernatant fractions from each mutant with antibodies specific Kgp. $2.5 \mu \mathrm{g}$ of cell lysate and $5 \mu \mathrm{g}$ of the supernatant were applied in these experiments. (C) The activity levels of Kgp in the cell lysate and culture supernatant fractions from each mutant. The white and black bars indicate the cell lysate and the culture supernatant, respectively. The data are the mean \pm S.D. of values from 6 independent experiments. ${ }^{*}, p<0.01$ for the indicated comparisons.

Fig. 4 - The activity and protein levels of Rgp in Kgp mutants with changed active-site Cys residues expressed in KDP129. (A) A Western blot analysis of the cell lysate and culture supernatant fractions from each mutant with antibodies reacting with Rgp and Kgp. $1 \mu \mathrm{g}$ of cell lysate and $2 \mu \mathrm{g}$ of the supernatant were applied in these experiments. (B) The activity levels of $\mathrm{Rgp}$ in the cell lysate and culture supernatant fractions from each mutant. The white and black bars indicate the cell lysate and the culture supernatant, respectively. The data are the mean \pm S.D. of values from 6 independent experiments. 
(A)

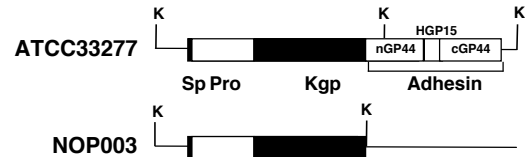

(B)

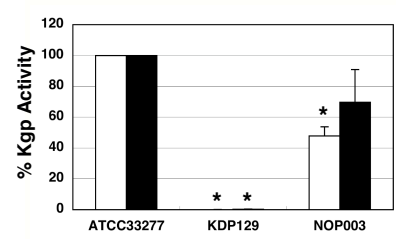

(D)

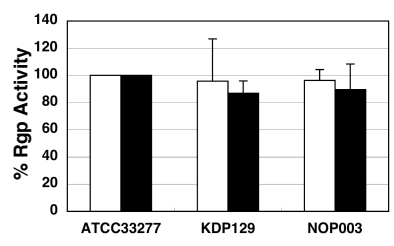

(C)

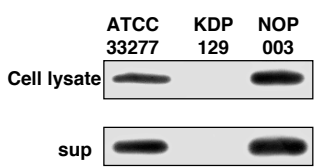

(E)

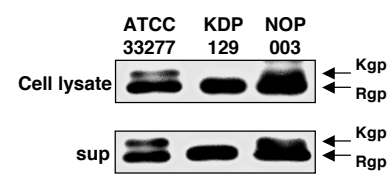

(A)

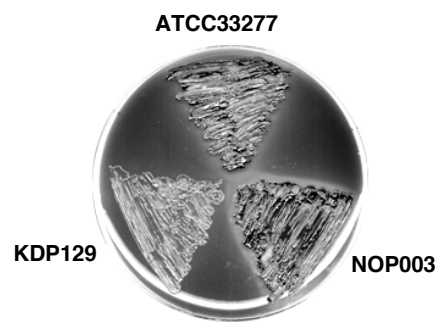

(B)

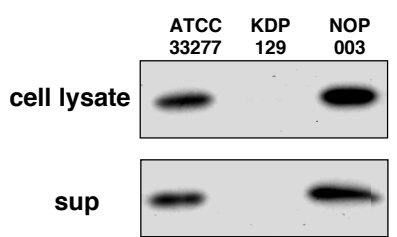

(A) GNCCVTAQFD ATCC33277 GNACVTAQFD NOP031 (C476A) GNCAVTAQFD NOP021 (C477A) GNAAVTAQFD NOP011 (C476A/C477A)

(B)

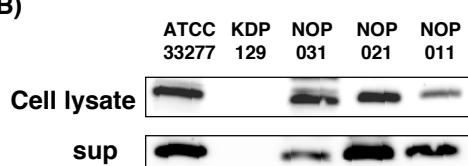

(C)

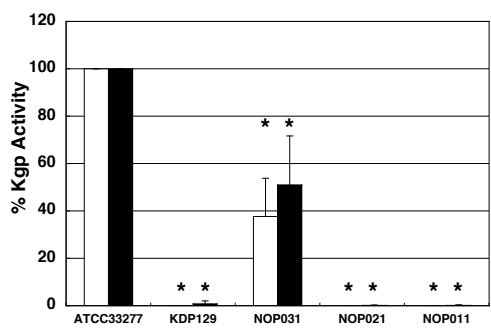

(A)

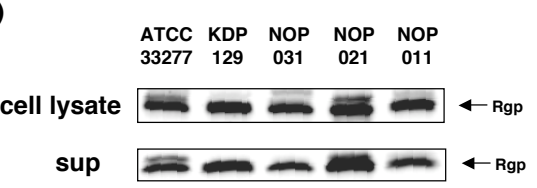

(B)

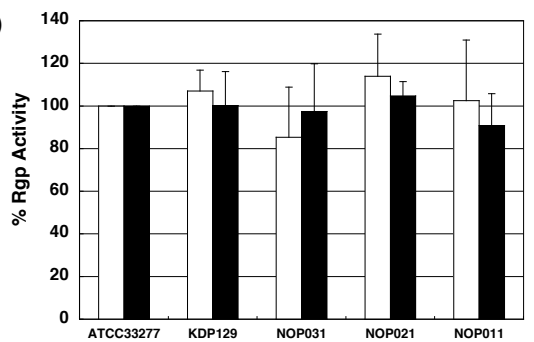

